\title{
A Smoking Gun in the Carina Nebula
}

\author{
Kenji Hamaguchi ${ }^{1,2}$, Michael F. Corcoran ${ }^{1,3}$, Yuichiro Ezoe ${ }^{4}$, Leisa Townsley ${ }^{5}$, Patrick \\ Broos $^{5}$, Robert Gruendl ${ }^{6}$, Kaushar Vaidya ${ }^{6, a}$, Stephen M. White ${ }^{7}$, Tod Strohmayer ${ }^{8}$, Rob \\ Petre $^{8}$, You-Hua $\mathrm{Chu}^{6}$
}

\begin{abstract}
The Carina Nebula is one of the youngest, most active sites of massive star formation in our Galaxy. In this nebula, we have discovered a bright X-ray source that has persisted for $\sim 30$ years. The soft X-ray spectrum, consistent with $k T \sim 128 \mathrm{eV}$ blackbody radiation with mild extinction, and no counterpart in the near- and mid-infrared wavelengths indicate that it is a $\sim 10^{6}$-year-old neutron star housed in the Carina Nebula. Current star formation theory does not suggest that the progenitor of the neutron star and massive stars in the Carina Nebula, in particular $\eta$ Car, are coeval. This result suggests that the Carina Nebula experienced at least two major episodes of massive star formation. The neutron star may be responsible for remnants of high energy activity seen in multiple wavelengths.
\end{abstract}

Subject headings: supernova remnants — stars: evolution — stars: formation — stars: neutron — ISM: bubbles - X-rays: stars

\section{Introduction}

Massive stars $\left(M \gtrsim 10 M_{\odot}\right)$ are born from giant molecular clouds along with many lower mass stars, forming a stellar cluster or association. Massive stars evolve orders of magnitude more quickly than lower mass stars, and die through supernova or hypernova explosions in $\lesssim 10^{7}$ years. High interstellar pressures that massive stars produce

\footnotetext{
${ }^{1}$ CRESST and X-ray Astrophysics Laboratory NASA/GSFC, Greenbelt, MD 20771

${ }^{2}$ Department of Physics, University of Maryland, Baltimore County, 1000 Hilltop Circle, Baltimore, MD 21250

${ }^{3}$ Universities Space Research Association, 10211 Wincopin Circle, Suite 500, Columbia, MD 21044

${ }^{4}$ Tokyo Metropolitan University, 1-1, Minami-Osawa, Hachioji, Tokyo, 192-0397, Japan

${ }^{5}$ Department of Astronomy and Astrophysics, Pennsylvania State University, 525 Davey Laboratory, University Park, PA 16802

${ }^{6}$ Department of Astronomy, University of Illinois, Urbana, IL 61801

${ }^{7}$ Department of Astronomy, University of Maryland, College Park, MD 20742

${ }^{8}$ Astrophysics Science Division, NASA Goddard Space Flight Center, Greenbelt, MD 20771

apreviously Kaushar Sanchawala
}

through their strong UV radiation and supernova explosions can compress a pre-existing cloud and trigger the formation of new stars (cf. Elmegreen 1998).

The Carina Nebula is one of the most massive star forming regions in our Galaxy. It contains two massive stellar clusters, Trumpler 14 and 16 (Figure 1), possessing over 50 massive stars with spectral types earlier than O6 ( $\gtrsim 40 M_{\odot}$, Smith 2006). The nebula is also home to one of the most massive stars in our Galaxy, $\eta$ Carinae, which has an estimated initial mass $\gtrsim 150 M_{\odot}$ and a current mass of about $90 M_{\odot}$ (Hillier et al. 2001).

At a distance of only $\sim 2.3 \mathrm{kpc}$ (Davidson \& Humphreys 1997), the Carina Nebula is one of the best sites for studying how very massive stars form and affect their environment. It shows signatures of violent activities: a bipolar supershell structure (Smith et al. 2000), strong turbulence in interstellar clouds (Yonekura et al. 2005), and hot $\mathrm{X}$-ray plasma through the entire nebula (Seward et al. 1979; Townsley 2006; Hamaguchi et al. 2007; Ezoe et al. 2008). Two primary mechanisms have been proposed to produce these structures. One is strong winds and UV radiation from massive stars 
in the nebula, while the other is supernova explosions. The energy budget and elemental abundance distribution favor the supernova mechanism (Yonekura et al. 2005; Hamaguchi et al. 2007). However, neither black hole, neutron star, nor clear remnant from a supernova, has been found in the Carina Nebula. In addition, any massive supernova progenitor is unlikely to be co-eval with the observed stars since it would be more massive than $\eta$ Car; this means that, if a supernova occurred in the Carina Nebula, its progenitor would probably have formed much earlier than $\eta$ Car. Thus, detection of any compact object in the Carina Nebula would be of vital interest for understanding the star formation history of the region.

From multiple sets of X-ray data, we found a promising neutron star candidate at the heart of the Carina Nebula. The source, which we call EHG7 (Ezoe et al. 2008), was detected as a very soft source in X-ray images $8.5^{\prime}$ southeast of $\eta$ Car, equivalent to $5.7 \mathrm{pc}$ in the projected physical distance assuming $d=2.3 \mathrm{kpc}$ (See Figure 1 ). The $\mathrm{X}$-ray source has also been reported contemporaneously in four other papers (Albacete Colombo et al. 2003; Ezoe et al. 2008; Pires \& Motch 2008; Pires et al. 2008), the last two also suggested EHG7 as a neutron star based on XMM-Newton and Chandra data. This paper presents a comprehensive study of the characteristics of EHG7 using all available X-ray data from Einstein and ROSAT observations to the latest XMM-Newton observations in 2009 to present conclusive evidence of the compact nature of EHG7.

\section{X-ray Observations and Analysis}

We found 32 X-ray observations with EHG7 in the field of view and spatial resolution better than $1^{\prime}$ in the HEASARC archive and our proprietary data (Table 1). Observations with lower spatial resolution (e.g. Einstein IPC, $A S C A$ ) failed to detect EHG7 due to severe contamination from surrounding soft diffuse X-ray emission. Throughout this document, individual observations with Einstein, ROSAT, XMM-Newton, and Chandra observations are designated EIN, ROS, XMM, CXO respectively, subscripted with the year, month, and day of the start time of the observations.

We used the software package SAS, version 8.0.1 for analysis of the XMM-Newton EPIC data
(Strüder et al. 2001). We followed the standard method $^{1}$ in data processing — screening out high background periods, selecting photon events and generating spectral response files. For the timing and spectral analysis, we took source events from an encircled region with $30^{\prime \prime}$ radius and background events from an annulus region with $60^{\prime \prime}$ outer and $30^{\prime \prime}$ inner radii. For data sets with the source close to 2 CCD gaps (see Table 1), source events are collected only from a CCD chip with the source peak. Comparison of these spectra with spectra of the other cameras in the same observation looked consistent, but we did not use them for flux measurements.

For source position determination on the Chandra image, we used a custom analysis method for Chandra data sets developed by Broos et al. (2007) and Broos et al. (in prep). In producing the Chandra spectral and timing data sets, we analyzed level 2 event data using the software package CIAO, version 4.0. We took source events from an encircled region with a $8^{\prime \prime}$ radius and background events from an annulus region with $16^{\prime \prime}$ outer and $8^{\prime \prime}$ inner radii.

For general light curve and spectral analysis of the above data sets and for the ROSAT and Einstein data processing, we used the software package HEAsoft version 6.5.1. We started from the pipeline products of the ROSAT and Einstein data. For the ROSAT image analysis, we excluded PHA channels above 10 to reduce particle background events for the HRI and extracted events between $0.3-2 \mathrm{keV}$ for the PSPC. For the PSPC spectral and timing analysis, we took source events from an encircled region with $30^{\prime \prime}$ radius and background events from an annulus region with $60^{\prime \prime}$ outer and 30" inner radii. For Einstein HRI calibration information, we referred the mirror vignetting at the location of EHG7 in $\mathrm{EIN}_{781224}\left(8.5^{\prime}\right.$ off-axis) to Harnden et al. (1984) (91\% at $0.6 \mathrm{keV}$ ) and estimated encircled energy of the point spread function (PSF) within a $32^{\prime \prime} \times 32^{\prime \prime}$ box at $76 \%$ using a calibration observation of Cyg X-1 at a similar off-axis angle (10': the dataset H1956N35.xic). The dead time was negligible and therefore ignored.

${ }^{1}$ http://xmm.esac.esa.int/sas/8.0.0/documentation/threads/ 
TABLE 1

X-Ray Observations with EHG7 in the Field of View

\begin{tabular}{|c|c|c|c|c|c|c|}
\hline Abbreviation & Observation ID & Observation Start & Detector/Filter & $\begin{array}{l}\text { Offset } \\
(\operatorname{arcmin})\end{array}$ & $\begin{array}{l}\text { Exposure } \\
(\text { ksec })\end{array}$ & 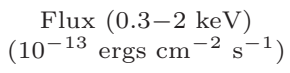 \\
\hline $\mathrm{EIN}_{781214}$ & 1074 & 1978 Dec. $14,05: 44$ & HRI-2 & 8.5 & 17.4 & $0.89 \pm 0.54$ \\
\hline $\mathrm{ROS}_{900727}$ & CA150037H.N1 & 1990 Jul. 27, 00:12 & HRI & 15.0 & 3.3 & $<1.1$ \\
\hline ROS $_{911215}$ & US200108P.N1 & 1991 Dec. 15, 9:58 & PSPCB & 19.4 & 1.6 & $<2.2$ \\
\hline $\operatorname{ROS}_{920612}$ & US900176P.N1 & 1992 Jun. 12, 22:33 & PSPCB & 8.7 & 23.6 & $0.94 \pm 0.16$ \\
\hline $\operatorname{ROS}_{920731}$ & WG900385H.N1 & 1992 Jul. 31, 01:03 & HRI & 8.7 & 11.4 & $1.04 \pm 0.33$ \\
\hline $\operatorname{ROS}_{920809}$ & WG201262P.N1 & 1992 Aug. 9, 22:21 & PSPCB & 13.8 & 5.7 & $1.43 \pm 0.47$ \\
\hline $\operatorname{ROS}_{920810}$ & WG200709P-1.N1 & 1992 Aug. 10, 19:03 & $\mathrm{PSPCB}$ & 35.4 & 5.9 & $<2.4$ \\
\hline $\operatorname{ROS}_{921215}$ & US900176P-1.N1 & 1992 Dec. $15,17: 39$ & PSPCB & 8.7 & 14.1 & $0.69 \pm 0.18$ \\
\hline $\operatorname{ROS}_{940106}$ & WG900385H-2.N1 & 1994 Jan $6,1: 54$ & HRI & 8.7 & 0.5 & $<7.7$ \\
\hline $\mathrm{ROS}_{940721}$ & WG900385H-3.N1 & 1994 Jul. 21, 02:04 & HRI & 8.7 & 40.1 & $0.91 \pm 0.18$ \\
\hline $\operatorname{ROS}_{960813}$ & US900644H.N1 & 1996 Aug. 13, 21:07 & HRI & 8.7 & 1.7 & $<3.3$ \\
\hline $\operatorname{ROS}_{971223}$ & US202331H.N1 & 1997 Dec. 23, 08:59 & HRI & 8.7 & 46.5 & $0.82 \pm 0.16$ \\
\hline $\mathrm{XMM}_{000726}$ & 112580601 & 2000 Jul. 26, 05:08 & MOS/thick & 8.7 & $-/ 33.2 / 30.1^{\dagger}$ & $1.04 \pm 0.18$ \\
\hline $\mathrm{XMM}_{000727}$ & 112580701 & 2000 Jul. 27, 23:58 & MOS/thick & 8.7 & $-/ 10.9 / 7.9^{\dagger}$ & $1.21 \pm 0.30$ \\
\hline $\mathrm{XMM}_{030125}$ & 145740101 & 2003 Jan. 25, 12:58 & MOS/thick & 8.4 & $-/ 6.9 / 6.9^{\dagger}$ & $1.40 \pm 0.36$ \\
\hline $\mathrm{XMM}_{030127 \mathrm{~A}}$ & 145740201 & 2003 Jan. 27, 01:04 & MOS/thick & 8.4 & $-/ 6.8^{\dagger} / 6.9^{\dagger}$ & $(1.21 \pm 0.25)$ \\
\hline $\mathrm{XMM}_{030129 \mathrm{~A}}$ & 145740401 & 2003 Jan. 29, 01:41 & MOS/thick & 8.4 & $-/ 8.4^{\dagger} / 8.4$ & $0.95 \pm 0.27$ \\
\hline $\mathrm{XMM}_{030129 \mathrm{~B}}$ & 145740501 & 2003 Jan. 29, 23:55 & MOS/thick & 8.4 & $-/ 6.9^{\dagger} / 6.9$ & $1.30 \pm 0.32$ \\
\hline $\mathrm{XMM}_{030608}$ & 160160101 & 2003 Jun. 08, 13:30 & MOS/thick & 8.4 & $-/ 17.0 / 16.5$ & $1.10 \pm 0.19$ \\
\hline $\mathrm{XMM}_{030613}$ & 160160901 & 2003 Jun. 13, 23:52 & MOS/thick & 8.4 & $-/ 31.1 / 31.1$ & $1.03 \pm 0.15$ \\
\hline $\mathrm{XMM}_{030722}$ & 145780101 & 2003 Jul. 22, 01:51 & MOS/thick & 8.4 & $-/ 8.4 / 8.4^{\dagger}$ & $1.19 \pm 0.34$ \\
\hline $\mathrm{XMM}_{030802}$ & 160560101 & 2003 Aug. 02, 21:01 & MOS2/thick & 8.4 & $-/-/ 11.9$ & $1.14 \pm 0.26$ \\
\hline $\mathrm{XMM}_{030809}$ & 160560201 & 2003 Aug. 09, 01:44 & MOS/thick & 8.4 & $-/ 12.2 / 12.2$ & $1.06 \pm 0.20$ \\
\hline $\mathrm{XMM}_{030818}$ & 160560301 & 2003 Aug. 18, 15:23 & MOS/thick & 8.4 & $-/ 18.5 / 18.5$ & $1.06 \pm 0.17$ \\
\hline $\mathrm{XMM}_{041207}$ & 206010101 & 2004 Dec. $7,7: 30$ & pn/medium & 16.3 & $19.4^{\dagger} /-/-$ & $(0.88 \pm 0.14)$ \\
\hline $\mathrm{XMM}_{060131}$ & 311990101 & 2006 Jan. $31,18: 04$ & pn\&MOS/thick & 8.7 & $25.2 / 65.3^{\dagger} / 65.3$ & $1.05 \pm 0.12$ \\
\hline $\mathrm{CXO}_{080905}$ & 9488 & 2008 Sep. 5, 21:24 & ACIS-I & 6.7 & 59.4 & $0.87 \pm 0.08$ \\
\hline $\mathrm{XMM}_{090105}$ & 560580101 & 2009 Jan 5, 10:23 & MOS/thick & 8.5 & $-/ 14.0 / 13.9$ & $1.17 \pm 0.19$ \\
\hline $\mathrm{XMM}_{090109}$ & 560580201 & 2009 Jan 9, 14:28 & MOS/thick & 8.5 & $-/ 11.4 / 11.4$ & $1.17 \pm 0.21$ \\
\hline $\mathrm{XMM}_{090115}$ & 560580301 & 2009 Jan $15,11: 23$ & MOS/thick & 8.5 & $-/ 25.4 / 25.4$ & $0.97 \pm 0.15$ \\
\hline $\mathrm{XMM}_{090202}$ & 560580401 & 2009 Feb 2, 04:46 & MOS2/thick & 8.5 & $-/-/ 26.2$ & $1.09 \pm 0.18$ \\
\hline
\end{tabular}

Note.-Abbreviation: EIN: Einstein, ROS: ROSAT, XMM: XMM-Newton, CXO: Chandra, Detector/Filter: optical blocking filter for XMM-Newton, Offset: angle of EHG7 from the nominal coordinate, Exposure: pn/MOS1/MOS2 for XMM-Newton, Flux: Error denotes the 90\% confidence range. The upper limit is at a $3 \sigma$ level. The flux errors include uncertainty of the absolute flux calibration of each telescope (Einstein, ROSAT, XMM-Newton: 10\%, Chandra: 5\%, ROSAT User's Handbook - http://heasarc.gsfc.nasa.gov/docs/rosat/ruh/handbook/handbook.html, Beuermann et al. (2006), XMM-SOC-CAL-TN-0018 — http://xmm2.esac.esa.int/external/xmm_sw_cal/calib/documentation/index.shtml\#EPIC, Calibration Requirements and Present Status — http://asc.harvard.edu/cal/docs/cal_present_status.html\#abs_eff). ${ }^{\dagger}$ the source peak was close to 2 CCD gaps or an edge of the field of view. Those data sets were not used for flux measurements in the right column. 

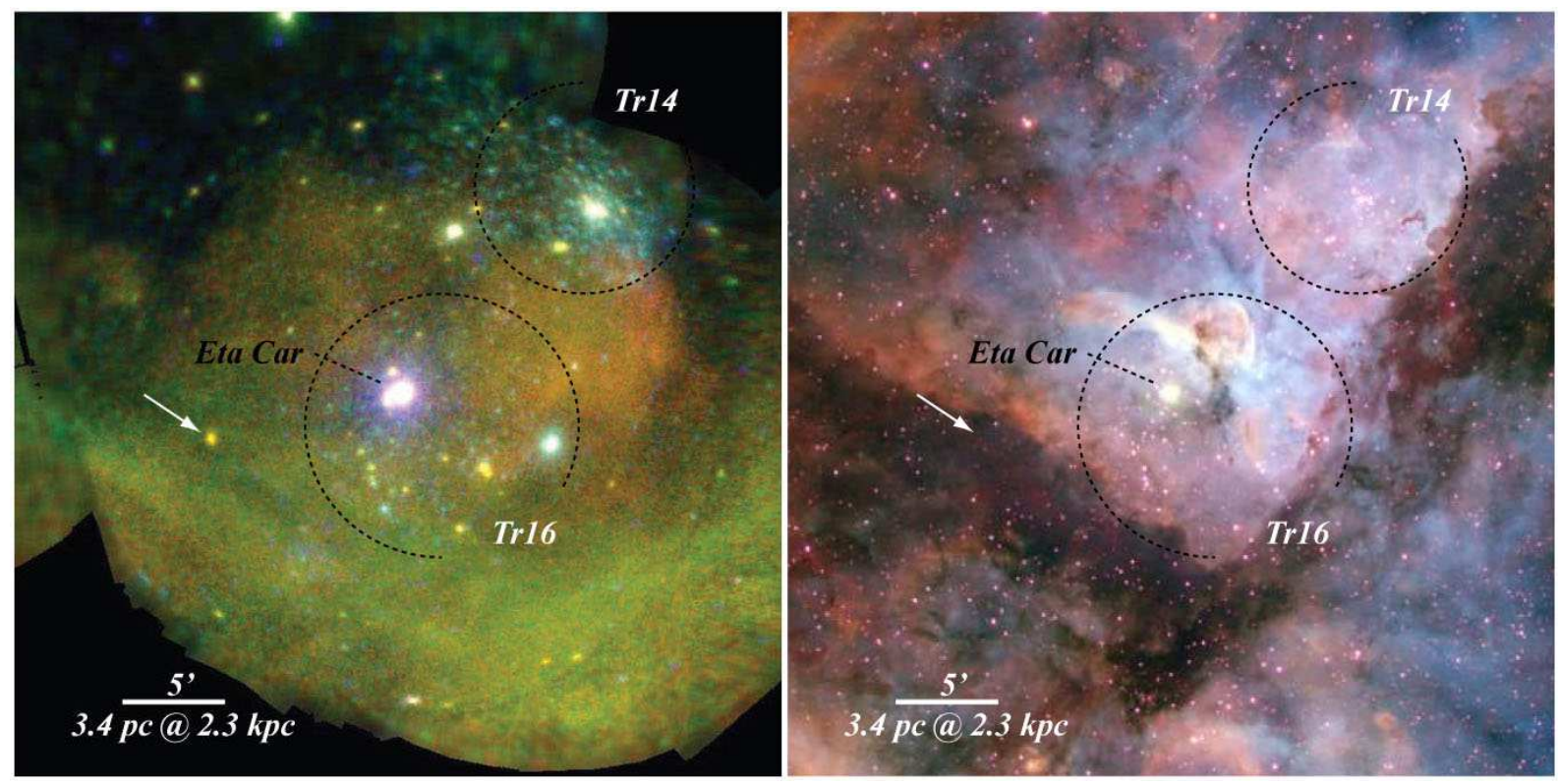

Fig. 1. - The central part of the Carina Nebula in X-rays taken with XMM-Newton (Left, red: $0.4-0.7 \mathrm{keV}$, green: $0.7-1.3 \mathrm{keV}$, blue: $2-7 \mathrm{keV}$ ) and in optical emission lines (Right, Credit: Nathan Smith, University of Minnesota/NOAO/AURA/NSF). EHG7, the source discussed here, is denoted by the white arrow. It is located on the conspicuous V-shaped dark lane running across the optical image and is surrounded by diffuse X-ray emission. The black dotted line and circles depict positions of the super massive star $\eta$ Car and massive stellar clusters, Trumpler 14 and 16, respectively.

\section{Results}

The Chandra observation $\mathrm{CXO}_{080905}$, performed as a part of a Very Large Project to map the Carina Nebula in X-rays (PI: Leisa Townsley), gives the most precise and reliable absolute position of EHG7 (Figure 2). In this observation, EHG7 was imaged $6.7^{\prime}$ off-axis on the ACIS-I detector. The absolute coordinates of the source were determined to be $\left(\alpha_{2000}, \delta_{2000}\right)=$ $\left(10^{\mathrm{h}} 46^{\mathrm{m}} 08.72^{\mathrm{s}},-59^{\circ} 43^{\prime} 06.5^{\prime \prime}\right)$ with less than $1^{\prime \prime}$ positional uncertainty after correcting the Chandra astrometry by cross correlation of X-ray and infrared sources from the 2MASS catalogue and correlating the PSF at the location of this source with the image of the source.

We searched for near- and mid-infrared counterparts from images obtained with the IRSF (InfraRed Survey Facility) 1.4m telescopes (see Sanchawala et al. 2007a,b, for details) and in all available Spitzer InfraRed Array Camera (IRAC; Fazio et al. 2004) observations combined, but these images showed no counterpart object within the er- ror circle of the X-ray source (Figure 2). We used standard aperture photometry routines in IRAF to estimate the 3- $\sigma$ upper-limits, which were $18.5 \mathrm{mag}, 19.5 \mathrm{mag}$ and $18.5 \mathrm{mag}$ in the $J, H$ and $K_{S}$ bands and $0.84,0.51,4.7$, and $13.6 \mathrm{mJy}$ in the IRAC 3.6, 4.5, 5.8, and $8.0 \mu \mathrm{m}$ bands, respectively. Assuming blackbody spectra with temperatures between 2000 and $10000 \mathrm{~K}$, the bolometric flux of EHG7 was limited to $<5 \times 10^{-13} \mathrm{ergs} \mathrm{cm}^{-2} \mathrm{~s}^{-1}$. We note that Pires et al. (2008) measured upper limits of $25^{m}$ in $R$ and $B$-band optical images.

$\mathrm{X}$-ray emission from EHG7 was detected at above $3 \sigma$ significance in all observations in Table 1 except for 5 ROSAT observations with short exposures. None of these observations showed any significant time variation from EHG7 at greater than $90 \%$ confidence in a fit of each light curve in the whole instrumental energy band with a constant flux model. For data sets with no spectral resolution and/or poor photon statistics, we measured the photon count rate or upper limit in whole band images using the sosta package in ximage, taking a suitable nearby source free region as background. 


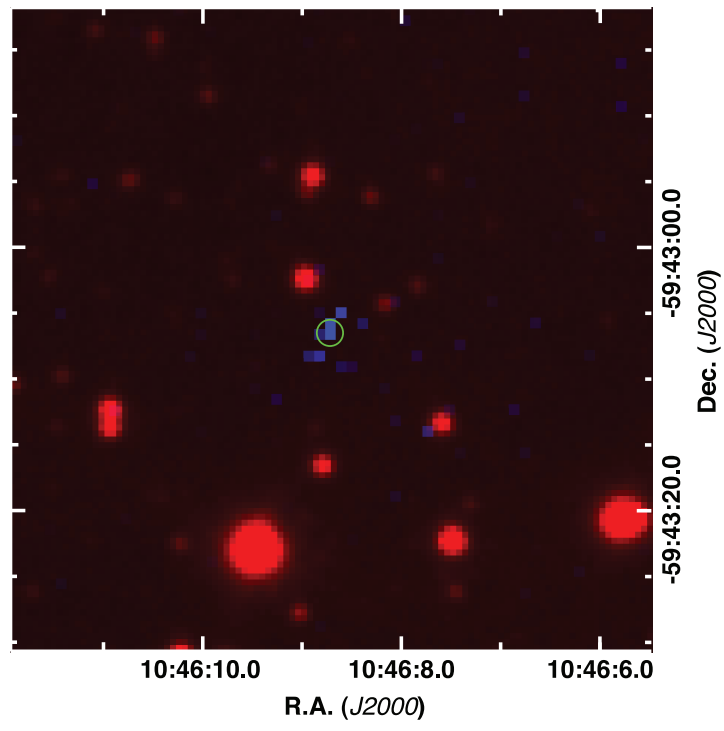

Fig. 2.- Magnified image of the neutron star field (blue: a Chandra X-ray image after the PSF image reconstruction, red: a deep $H$ band image taken with the IRSF observatory). The position of the $\mathrm{X}$-ray source EHG7 is marked with a green circle with a $1^{\prime \prime}$ radius; it has no infrared counterpart. Another X-ray source about $10^{\prime \prime}$ southwest from EHG7 has a counterpart in the $H$-band.

We then converted the source counts to energy flux using the PIMMS tool, assuming blackbody radiation with $k T \sim 128 \mathrm{eV}$ and $N_{\mathrm{H}} \sim 3.2 \times 10^{21} \mathrm{~cm}^{-2}$, derived from a best-fit model to the XMM-Newton spectrum as described below. For the other data sets, we measured source flux from time averaged spectra, assuming the same spectral model. The results are shown in Table 1. EHG7 did not show any significant flux variation above $\sim 30 \%$ between the observations though a formal fit of these data rejected a constant flux model at above $90 \%$ confidence $\left(\chi^{2} /\right.$ d.o.f $\left.=38.45 / 23\right)$. The $\mathrm{X}$-ray emission was stable for $\sim 30$ years with $F_{\mathrm{X}}(0.3-2 \mathrm{keV})$ $\approx 1.06 \times 10^{-13} \mathrm{ergs} \mathrm{cm}^{-2} \mathrm{~s}^{-1}$

Individual observations did not have sufficient photon statistics for detailed spectral analysis. Since the spectral shape did not change significantly between observations ${ }^{2}$, we combined all the

\footnotetext{
${ }^{2}$ We simultaneously fitted $0.3-3 \mathrm{keV}$ spectra of all the available EPIC cameras in each observation by an absorbed blackbody model and found that $95 \%$ confidence ranges of the $N_{\mathrm{H}}$ and $k T$ parameters overlapped with each other.
}

source spectra taken between 2000 and 2006 with the XMM-Newton MOS and pn detectors (Figure 3). We used the HEAsoft tool mathpha to combine the source and background spectra, marfrmf to merge arf and rmf files and then addrmf to average produced response files over by weighting them by net source counts. The combined $\mathrm{X}$-ray spectrum showed significant emission only below $2 \mathrm{keV}$ and is almost featureless except for a slight dip at around $0.9 \mathrm{keV}$ and a strong dip at around $0.6 \mathrm{keV}$. The latter feature at $\sim 0.6 \mathrm{keV}$ was perhaps produced by edge absorption of oxygen in the interstellar medium and in the CCD detector response. The spectrum can be reproduced using a 1-temperature optically-thin thermal plasma emission model suffering absorption by neutral gas along the line of sight (Table 2), but the best-fit model has an unusually small elemental abundance of $\lesssim 10^{-3}$ solar, without evidence of emission lines from oxygen, iron, or neon atoms near $0.5-1 \mathrm{keV}$. The spectrum also can be fit by a 1-temperature blackbody emission model with a temperature of $128 \pm 7 \mathrm{eV}$ (90\% confidence) suffering absorption by neutral gas with hydrogen column density of $3.2 \pm 0.4 \times 10^{21} \mathrm{~cm}^{-2}$ (90\% confidence). The spectrum could not be adequately fit using an absorbed power law model. We note that Pires et al. (2008) derived a similar best-fit result in individual fits of XMM-Newton spectra.

The hydrogen column density is consistent with interstellar absorption to the Carina Nebula $\left(\sim 3 \times 10^{21} \mathrm{~cm}^{-2}\right.$, see discussion in Leutenegger et al. 2003). The $90 \%$ confidence upper limit $\left(3.6 \times 10^{21} \mathrm{~cm}^{-2}\right)$ is much smaller than absorption through our Galaxy $\left(\sim 1.2 \times 10^{22} \mathrm{~cm}^{-2}\right.$, Kalberla et al. 2005), which emission from an AGN should suffer. It is also significantly smaller than absorption to the background stars in the field, which have visual extinctions twice as large as Carina cluster members (DeGioia-Eastwood et al. 2001). This result suggests that EHG7 is in front of the molecular cloud that lies behind the Trumpler 14 and 16 clusters, and neighbors the Carina Nebula.

The ratio of the X-ray flux to the bolometric flux, $\log F_{\mathrm{X}} / F_{\text {bol }}>-0.7$, was significantly larger than that typically seen in stellar X-ray emission $(\lesssim-3$, Gagné et al. 1995). This large $F_{\mathrm{X}} / F_{\text {bol }}$ means that EHG7 is unlikely to be a star in the Carina star-forming complex nor in the foreand background but must be a compact object 
TABLE 2

Spectral Fits

\begin{tabular}{cccccc}
\hline \hline \multicolumn{1}{c}{ Model } & $\begin{array}{c}k T \\
(\mathrm{eV})\end{array}$ & $\begin{array}{c}\text { Abundance } \\
(\text { solar })\end{array}$ & $\begin{array}{c}N_{\mathrm{H}} \\
\left(10^{21} \mathrm{~cm}^{-2}\right)\end{array}$ & $\begin{array}{c}L_{\mathrm{X}} \\
\left(10^{32} \mathrm{ergs} \mathrm{s}^{-1}\right)\end{array}$ & $\Delta \chi^{2}$ (d.o.f) \\
\hline 1 T APEC & $171(162-174)$ & $<8.0 \times 10^{-4}$ & $5.4(5.0-5.8)$ & 32 & $1.51(125)$ \\
1 T Blackbody & $128(121-133)$ & $\cdots$ & $3.2(2.8-3.6)$ & 4.6 & $1.49(126)$ \\
\hline
\end{tabular}

Note.-Absorption corrected $L_{\mathrm{X}}$ between $0.3-8 \mathrm{keV}$ assuming $d=2.3 \mathrm{kpc}$. The parentheses in the $k T$ and $N_{\mathrm{H}}$ columns denote the $90 \%$ confidence range.

such as a black hole, a white dwarf or a neutron star. The X-ray characteristics of this source, a single temperature blackbody spectrum without time variation longer than hourly timescales, are typical of isolated neutron stars, where the emission comes from the cooling neutron star surface. It is unlikely to be a black hole, which would show strong time variation on both short and long timescales, reflecting activity around the accretion disk near the event horizon. It is also unlikely to be a white dwarf, which has normally significantly lower blackbody temperatures than $100 \mathrm{eV}$ (Kahabka \& van den Heuvel 1997). Although some white dwarfs with masses close to the Chandrasekhar limit can have $k T \gtrsim 100 \mathrm{eV}$, they should be more than five orders of magnitudes more luminous (Sala \& Hernanz 2005). All this evidence shows that the source is most likely a neutron star.

We found no radio counterpart of EHG7 in a continuum radio map of the Carina region from the Southern Galactic Plane Survey (McClureGriffiths et al. 2005), but the resolution of these data is only $1^{\prime}$ and weak point sources will not be detected against the strong nebular radio sources Car I and Car II. The ATNF Pulsar Catalogue (Manchester et al. 2005) lists 11 pulsars within $2^{\circ}$ of EHG7, but no source coincident with EHG7 itself. We searched for a pulse from the XMMNewton pn data between $0.3-2 \mathrm{keV}$ in $\mathrm{XMM}_{060131}$ using the Fast Fourier Transform and $\mathrm{Z}^{2}$ searches. However, we detected no significant pulses from EHG7 in the X-ray data in the frequency range between $10^{-4}-13.6 \mathrm{~Hz}$. A 90\% confidence upper limit on any signal amplitude in this frequency range was $27.7 \%$ (rms).

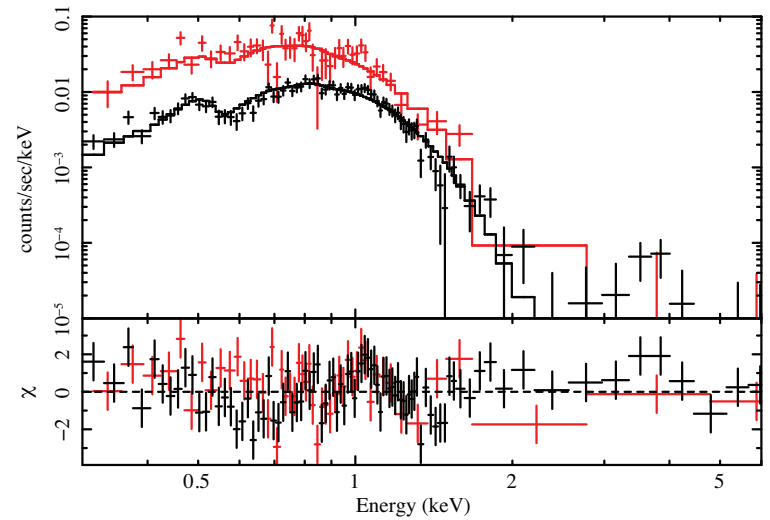

Fig. 3.- Top panel : XMM-Newton MOS (black) and pn $(r e d)$ spectra combined from all the available data. The solid line shows the best-fit model of the spectra between $0.3-3 \mathrm{keV}$ by an absorbed blackbody model. Bottom panel : the residuals of the $\chi^{2}$ fit.

\section{Discussion}

The intrinsic X-ray luminosity is $4.6 \times 10^{32} \mathrm{ergs} \mathrm{s}^{-1}$ or equivalent to an emitting radius of $\sim 7.3 \mathrm{~km}$ assuming $k T=128 \mathrm{eV}$ and $d=2.3 \mathrm{kpc}$. Assuming the standard cooling curve of a $M=1.4 M_{\odot}$ neutron star (Tsuruta 2006), the age of EHG7 is estimated at $\sim 0.5-1 \times 10^{6}$-year. The blackbody temperature, $0.13 \mathrm{keV}$, is also typical of neutron stars older than 1000 years (e.g. 1E1207.4-5209, PSR B0656+14 and PSR B1055-52, Sanwal et al. 2002; De Luca et al. 2005). The X-ray spectrum did not show a non-thermal power-law component in the hard band that would typically originate from charged particles in the magnetosphere. The upper limit of $L_{\mathrm{X}}[0.5-10 \mathrm{keV}] \lesssim 5 \times 10^{30} \mathrm{ergs} \mathrm{s}^{-1}$ estimated from a fit of the spectrum above $2 \mathrm{keV}$ 
assuming an additional power-law component with a fixed $\Gamma=1.7$ is similarly lower than is typical for middle-aged rotation-powered pulsars of a few $\times 10^{5}$ year old (De Luca et al. 2005; Manzali et al. 2007). These results suggest that the neutron star originated in a supernova explosion about $10^{6}$ years ago.

The dip feature at $\sim 0.9 \mathrm{keV}$ can be produced by fundamental cyclotron absorption by electrons in the magnetic field with $\sim 7.5 \times 10^{10}$ Gauss, which is typical of middle-aged radio pulsars (e.g. Reisenegger 2001), or by protons in the magnetic fields with $\sim 10^{14}$ Gauss, reminiscent of the $\mathrm{X}$ ray Dim Isolated Neutron Star (XDINS, see e.g. Haberl 2007; Kaplan 2008). These stars have short pulsation periods of $\sim$ a few hundred millisecond and/or small pulse fraction of $<20 \%$. The existing X-ray data sets do not have enough time resolution nor photon statistics to detect such a pulse.

Two other neutron stars have been discovered near other star forming regions (Figer et al. 2005; Muno et al. 2006). Both of them have characteristics of magnetars - neutron stars with strong magnetic fields up to $10^{15}$ Gauss - whose progenitor exploded within the last $\sim 10^{4}$ years and who might represent the high mass end of stars born in these star forming regions. However, the neutron star discovered in the Carina Nebula cannot be co-eval with the current generation of stars. Since the higher mass stars evolve faster, the progenitor of the neutron star would have to be more massive than $\eta$ Car. Eta Car's estimated initial mass $\gtrsim 150 M_{\odot}$ (Hillier et al. 2001) is much higher than the conventional progenitor mass of neutron stars (less than $\sim 25 M_{\odot}$ ) and explosion of such a massive star would likely produce a black hole, instead of a neutron star although Woosley et al. (2002) found that explosions of stars with masses up to $\sim 60 M_{\odot}$ might produce a neutron star under certain circumstances.

Based on standard stellar evolution models, the progenitor of the neutron star should have been born $6-30 \times 10^{6}$ year ago with an initial mass between $8-25 M_{\odot}$. This is significantly earlier than formation of the massive stars in the Trumpler 14 and 16 clusters which occurred less than $3 \times 10^{6}$ years ago (DeGioia-Eastwood et al. 2001; Massey et al. 2001). This suggests that the Carina Nebula has experienced at least 2 episodes of star forma- tion. This is consistent with stellar population studies (DeGioia-Eastwood et al. 2001) which also suggest that intermediate-mass stars have formed continuously over the last $10^{7}$ years. An alternative scenario is that EHG7 escaped from a nearby star forming region, coming close to the Carina nebula by chance. One possible site is the Carina flare region located at $\sim 2-10^{\circ}$ above the Carina nebula in Galactic coordinates. This region is suspected to have experienced major star forming activity over $2 \times 10^{7}$ yr (Fukui et al. 1999). Another possibility is that EHG7 thermalizes through Bondi-Hoyle-Lyttleton accretion from the nebular gas (Blaes \& Madau 1993). In that case, it can be much older than $10^{6}$ yr and its birth place is unpredictable.

The last episode of star formation in the Carina Nebula appears to have been nearly contemporaneous with the supernova explosion of the progenitor of this neutron star. This may suggest that the expanding H II region, wind-blown bubble and/or supernova explosion of this star (and perhaps others yet undetected) played a role in triggering the last episode of star formation. Although the neutron star is currently well away from the nebula center, it could have moved the projected distance in $\sim 10^{6}$ year with a transverse velocity of only $\sim 6 \mathrm{~km} \mathrm{~s}^{-1}$, which is much lower than typical kick velocities of radio pulsars (several hundred $\mathrm{km} \mathrm{s}^{-1}$, Lyne \& Lorimer 1994).

Discovery of a neutron star supports the argument that the diffuse high energy emission observed in the Carina Nebula originated in an ancient supernova explosion. Some measurements require multiple supernova explosions in this field (Yonekura et al. 2005; Hamaguchi et al. 2007), so that the neutron star may be one of many neutron stars and black holes hidden in the Carina Nebula. If the soft extended X-ray plasma in reality heated up by supernova explosions $\sim 10^{6}$ years ago, it would represent an important phase of the evolution of inter stellar medium when hot gas produced by supernova remnants merge together to form the hot ionized intercloud medium (HIM) component (McKee \& Ostriker 1977). This result may have implications for the origin of diffuse Xray emission observed now from many star forming regions (Townsley et al. 2003; Güdel et al. 2008).

We are grateful to T.R. Gull, K.E. Nielsen, 
S. Drake, K. Mukai, M. Ishida, C. Markwardt and anonymous referee for useful comments. This work is performed while K.H. was supported by the NASA Astrobiology Program under CAN 03-OSS-02. This research has made use of data and softwares obtained from the High Energy Astrophysics Science Archive Research Center (HEASARC), provided by NASA's Goddard Space Flight Center and Chandra X-ray Center (CXC).

Facilities: CXO (ACIS-I), XMM-Newton (EPIC), ROSAT (PSPC, HRI), EINSTEIN (HRI) Spitzer (IRAC), IRSF

\section{REFERENCES}

Albacete Colombo, J. F., Méndez, M., \& Morrell, N. I. 2003, MNRAS, 346, 704

Blaes, O., \& Madau, P. 1993, ApJ, 403, 690

Beuermann, K., Burwitz, V., \& Rauch, T. 2006, A\&A, 458, 541

Broos, P. S., Feigelson, E. D., Townsley, L. K., Getman, K. V., Wang, J., Garmire, G. P., Jiang, Z., \& Tsuboi, Y. 2007, ApJS, 169, 353

Davidson, K., \& Humphreys, R. M. 1997, ARA\&A, 35, 1

De Luca, A., Caraveo, P. A., Mereghetti, S., Negroni, M., \& Bignami, G. F. 2005, ApJ, 623, 1051

DeGioia-Eastwood, K., Throop, H., Walker, G., \& Cudworth, K. M. 2001, ApJ, 549, 578

Elmegreen, B. G. 1998, in Astronomical Society of the Pacific Conference Series, Vol. 148, Origins, ed. C. E. Woodward, J. M. Shull, \& H. A. Thronson, Jr., 150

Ezoe, Y., Hamaguchi, K., Gruendl, R. A., Chu, Y.-H., Petre, R., \& Corcoran, M. F. 2008, PASJ, in print (astro-ph/0809.3495)

Fazio, G. G., and 64 others, 2004, ApJS, 154, 10

Figer, D. F., Najarro, F., Geballe, T. R., Blum, R. D., \& Kudritzki, R. P. 2005, ApJ, 622, L49

Fukui, Y., Onishi, T., Abe, R., Kawamura, A., Tachihara, K., Yamaguchi, R., Mizuno, A., \& Ogawa, H. 1999, PASJ, 51, 751
Gagné, M., Caillault, J. P., \& Stauffer, J. R. 1995, ApJ, 445, 280

Güdel, M., Briggs, K. R., Montmerle, T., Audard, M., Rebull, L., \& Skinner, S. L. 2008, Science, 319,309

Haberl, F. 2007, Ap\&SS, 308, 181

Hamaguchi, K., and 11 others, 2007, PASJ, 59, 151

Harnden, Jr., F. R., Fabricant, D. G., Harris, D. E., \& Schwarz, J. 1984, SAO Special Report, 393

Hillier, D. J., Davidson, K., Ishibashi, K., \& Gull, T. 2001, ApJ, 553, 837

Kahabka, P., \& van den Heuvel, E. P. J. 1997, ARA\&A, 35, 69

Kalberla, P. M. W., Burton, W. B., Hartmann, D., Arnal, E. M., Bajaja, E., Morras, R., \& Pöppel, W. G. L. 2005, A\&A, 440, 775

Kaplan, D. L. 2008, 40 Years of Pulsars: Millisecond Pulsars, Magnetars and More, ed. C. Bassa, Z. Wang, A. Cumming, \& V. M. Kaspi, 983, 331

Leutenegger, M. A., Kahn, S. M., \& Ramsay, G. 2003, ApJ, 585, 1015

Lyne, A. G., \& Lorimer, D. R. 1994, Nature, 369, 127

Manchester, R. N., Hobbs, G. B., Teoh, A., \& Hobbs, M. 2005, AJ, 129, 1993

Manzali, A., De Luca, A., \& Caraveo, P. A. 2007, ApJ, 669, 570

Massey, P., DeGioia-Eastwood, K., \& Waterhouse, E. 2001, AJ, 121, 1050

McClure-Griffiths, N. M., Dickey, J. M., Gaensler, B. M., Green, A. J., Haverkorn, M., \& Strasser, S. 2005, ApJS, 158, 178

McKee, C. F., \& Ostriker, J. P. 1977, ApJ, 218, 148

Muno, M. P., and 11 others, 2006, ApJ, 636, L41 
Pires, A. M., \& Motch, C. 2008, in American Institute of Physics Conference Series, Vol. 983, 40 Years of Pulsars: Millisecond Pulsars, Magnetars and More, ed. C. Bassa, Z. Wang, A. Cumming, \& V. M. Kaspi, 363-365

Pires, A. M., Motch, C., Turolla, R., Treves, A., \& Popov, S. B. 2008, A\&A, accepted (astro$\mathrm{ph} / 0812.4151)$

Reisenegger, A. 2001, in Astronomical Society of the Pacific Conference Series, Vol. 248, Magnetic Fields Across the Hertzsprung-Russell Diagram, ed. G. Mathys, S. K. Solanki, \& D. T. Wickramasinghe, 469

Sala, G., \& Hernanz, M. 2005, A\&A, 439, 1061

Sanchawala, K., Chen, W.-P., Lee, H.-T., Chu, Y.-H., Nakajima, Y., Tamura, M., Baba, D., \& Sato, S. 2007a, ApJ, 656, 462

Sanchawala, K., and 8 others, 2007b, ApJ, 667, 963

Sanwal, D., Pavlov, G. G., Zavlin, V. E., \& Teter, M. A. 2002, ApJ, 574, L61

Seward, F. D., Forman, W. R., Giacconi, R., Griffiths, R. E., Harnden, F. R. J., Jones, C., \& Pye, J. P. 1979, ApJ, 234, L55

Smith, N. 2006, MNRAS, 367, 763

Smith, N., Egan, M. P., Carey, S., Price, S. D., Morse, J. A., \& Price, P. A. 2000, ApJ, 532, L145

Strüder, L., et al. 2001, A\&A, 365, L18

Townsley, L. K. 2006, in the STScI May Symposium, "Massive Stars: From Pop III and GRBs to the Milky Way, ed. M. Livio, (astro$\mathrm{ph} / 0608173)$

Townsley, L. K., Feigelson, E. D., Montmerle, T., Broos, P. S., Chu, Y.-H., \& Garmire, G. P. 2003, ApJ, 593, 874

Tsuruta, S. 2006, in American Institute of Physics Conference Series, Vol. 847, Origin of Matter and Evolution of Galaxies, ed. S. Kubono, W. Aoki, T. Kajino, T. Motobayashi, \& K. Nomoto, 163-170
Woods, P. M., \& Thompson, C. 2006, in Compact stellar X-ray sources, ed. W. H. G. Lewin \& M. van der Klis (Cambridge: Cambridge Univ. Press)

Woosley, S. E., Heger, A., \& Weaver, T. A. 2002, Reviews of Modern Physics, 74, 1015

Yonekura, Y., Asayama, S., Kimura, K., Ogawa, H., Kanai, Y., Yamaguchi, N., Barnes, P. J., \& Fukui, Y. 2005, ApJ, 634, 476

This 2-column preprint was prepared with the AAS LATEX macros v5.2. 